\title{
A Fast Extraction Method in the Applicaton of UHV Transmission Line Fault Location*
}

\author{
Li Wang ${ }^{1,2}$, Jiale Suonan ${ }^{1}$, Zaibin Jiao ${ }^{1}$ \\ ${ }^{1}$ Department of Electrical Engineering, Xi'an Jiaotong University, Xi'an, China \\ ${ }^{2}$ Department of Technology Center, XJ Group Limited Company, Xuchang, China \\ Email: xiesley_ann@163.com, suonan@263.net, jiaozaibin@mail.xjtu.edu.cn
}

Received February, 2013

\begin{abstract}
To aim at the distribution parameter characteristics of UHV transmission line, this paper presents a fast extraction method (FE) to extract the accurate fundamentals of current and voltage from the UHV transmission line transient process, and locates the fault by utilizing two-end unsynchronized algorithm. The simulation result shows that this method has good performance of accuracy and stability, and has better location precision by comparing with results of one cycle Fourier algorithm. Therefore the method can efficiently improve the precision of fault location during the transient process, and makes the error of location results less than $0.5 \%$.
\end{abstract}

Keywords: UHV; Fast Extraction Method; Matrix Pencil; Transient Process; Fault Location

\section{Introduction}

Recently, with the development of communication technology and the improvement of electric power automation system, two-terminal fault location method [1-6] has been gradually popularized in the power system. With the increasing of UHV long transmission line, two-terminal fault location method that employs the accurate distributed parameter model and need not synchronized data of two-terminal will be applied extensively. Compared with HV and EHV transmission line, UHV transmission line has the characteristics of large transmission capacity, long transmission distance, smaller wave impedance and larger distributed capacitance, so the fault transient process will be more complicated, and accurately acquiring of fault electric quantity would be difficult. Most of existing location algorithms based on phasor method. In the transient process of UHV AC transmission system, the decaying DC offset of fault current and the decaying DC offset of fault voltage introduced by CVT extend the convergence time of the traditional Fourier algorithm, decrease the accuracy of algorithm, and also reduce the precision of electric quantities in the fault transient process.

In order to remove the influence of decaying DC offset, many domestic and foreign scholars have proposed some

\footnotetext{
"The research presented in this paper is supported by National Natural Science Foundation of China (51037005) and Specialized Research Fund for the Doctoral Program of Higher Education (2011020111 0056).
}

improved algorithms, such as difference Fourier algorithm[7] and parallel compensation method [8]. Although difference Fourier algorithm can suppress the DC offset in a certain extent, it cannot remove the decaying DC offset; meanwhile it also amplifies the content of harmonics. Parallel compensation method required the prior knowledge regarding the time constant of the DC offset, so it is difficult to realize in practical engineering. On the premise of relay protection rapid action, the present algorithm has a high demand of rapidity, which will sacrifice some accuracy and stability. But the UHV transmission line require not only the higher accuracy of fault location, but also protection quick trip over ten milliseconds, then the voltage and current data window would less than one cycle. In order to calculate fault location fast and accurately, it is important to introduce a new phasor extraction algorithm.

Based on Matrix Pencil method [9-12], the Fast Extraction method [13] is established by using matrix similar transformation and QR Factorization. It can quickly extract the fundamental frequency component of UHV transmission line, and remove the effect of decaying DC offset and high order harmonic component of the transient process. This paper uses the Fast Extraction method to identify the fundamental component of fault component within $20 \mathrm{~ms}$, and applies the fundamental to two-terminal fault location scheme for fault location, which greatly improves the accuracy of fault location during transient process, and has a good application prospect. 


\section{A Brief Introduction to Fast Extraction Method}

When faults occurred in UHV transmission line, fault current consists of a fundamental frequency, a decaying DC offset, and decaying harmonics. Capacitive voltage transformers produce low-frequency transient components having over damped behavior, which resemble DC offset components. So the fault voltage has the same frequency components as the fault current. Therefore the fault signal can be expressed as

$$
\begin{aligned}
i(t)= & A_{1} \cos \left(\omega_{1} t+\varphi_{1}\right)+\sum_{k=2}^{q} A_{k} e^{-\alpha_{k} t} \cos \left(\omega_{k} t+\varphi_{k}\right) \\
& +A_{q+1} e^{-\alpha_{q+1} t} .
\end{aligned}
$$

In (1), $A_{k}$ is the amplitude, and $\varphi_{k}$ is the phase, and $\alpha_{k} \quad\left(\alpha_{k} \geq 0\right)$ is the decaying factor.

Since we know that

$$
\cos \theta=\frac{e^{j \theta}+e^{-j \theta}}{2}
$$

then $i(t)$ can be expressed as

$$
i(t)=\frac{1}{2} \sum_{k=1}^{q+1} e^{-\alpha_{k} t}\left(A_{k} e^{j \varphi_{k}} e^{j \omega_{k} t}+A_{k} e^{-j \varphi_{k}} e^{-j \omega_{k} t}\right)
$$

where $\alpha_{1}=0, \omega_{q+1}=0$, and $\varphi_{q+1}=0$, other definitions are the same as in (1).

$$
\text { Let } p_{2 k-1}=\frac{A_{k} e^{j \varphi_{k}}}{2}, \varphi_{2 k-1}=-\alpha_{k}+j \omega_{k}, p_{2 k}=\frac{A_{k} e^{-j \varphi_{k}}}{2} \text {, }
$$
and $\varphi_{2 k}=-\alpha_{k}-j \omega_{k}$, then (3) can be expressed as

$$
i(t)=\sum_{k=1}^{M} p_{k} e^{\varphi_{k} t}
$$

where $M=2(p+1)$.

Since, in practice, one almost always deals with a discrete set of sampled transient data, (4) can be expressed as

$$
i\left(t_{n}\right)=i(n)=\sum_{k=1}^{M} p_{k} e^{\varphi_{k} \cdot n \Delta t}=\sum_{k=1}^{M} p_{k} z_{k}^{n}
$$

where $t_{n}=n \Delta t, \quad z_{k}=e^{\varphi_{k} \Delta t}$ and $\Delta t$ is the size of the time-stepping interval used in obtaining the sampled data.

In order to calculate the fundamental component of the input signal, as we all know, the fundamental amplitude is not decay, so let the reference signal $u(t)$ is as follows

$$
u(t)=\cos \omega_{1} t
$$

where $\omega_{1}=2 \pi 50 \mathrm{rad} / \mathrm{s}$.

So its discrete expression is as follows

$$
u(n)=\sum_{k=1}^{M} p_{k}^{\prime} z_{k}^{n}
$$

where $p_{1}^{\prime}=p_{2}^{\prime}=0.5, p_{3}^{\prime}=p_{4}^{\prime}=\cdots=p_{M}{ }^{\prime}=0$.

According to the Matrix Pencil method, we define two $(N-L+1) \times L$ rank matrices

$$
\begin{gathered}
{[I]=\left(\begin{array}{cccc}
i(0) & i(1) & \cdots & i(L-1) \\
i(1) & i(2) & \cdots & i(L) \\
\vdots & \vdots & \ddots & \vdots \\
i(N-L) & i(N-L+1) & \cdots & i(N-1)
\end{array}\right)} \\
{[U]=\left(\begin{array}{cccc}
u(0) & u(1) & \cdots & u(L-1) \\
u(1) & u(2) & \cdots & u(L) \\
\vdots & \vdots & \ddots & \vdots \\
u(N-L) & u(N-L+1) & \cdots & u(N-1)
\end{array}\right)}
\end{gathered}
$$

where $N / 2 \leq L \leq 2 N / 3$.

From the definitions of measured signal and referenced signal, then

$$
\begin{aligned}
& {[I]=\left[Z_{1}\right][P]\left[Z_{2}\right]} \\
& {[U]=\left[Z_{1}\right]\left[P^{\prime}\right]\left[Z_{2}\right]}
\end{aligned}
$$

where

$$
\begin{gathered}
{\left[Z_{1}\right]=\left(\begin{array}{cccc}
1 & 1 & \cdots & 1 \\
z_{1} & z_{2} & \cdots & z_{M} \\
\vdots & \vdots & \ddots & \vdots \\
z_{1}^{(N-L)} & z_{2}^{(N-L)} & \cdots & z_{M}^{(N-L)}
\end{array}\right)} \\
{\left[Z_{2}\right]=\left(\begin{array}{cccc}
1 & z_{1} & \cdots & z_{1}^{(L-1)} \\
1 & z_{2} & \cdots & z_{2}^{(L-1)} \\
\vdots & \vdots & \ddots & \vdots \\
1 & z_{M} & \cdots & z_{M}^{(L-1)}
\end{array}\right)} \\
{[P]=\operatorname{diag}\left[p_{1}, p_{2}, \cdots, p_{M}\right]} \\
{\left[P^{\prime}\right]=\operatorname{diag}\left[p_{1}^{\prime}, p_{2}^{\prime}, \cdots, p_{M}{ }^{\prime}\right]}
\end{gathered}
$$

Left multiplying $[U]$ by the pseudo-inverse of $[I]$ leads to

$$
\begin{aligned}
{[I]^{+}[U] } & =\left[Z_{2}\right]^{+}[P]^{-}\left[Z_{1}\right]^{+}\left[Z_{1}\right]\left[P^{\prime}\right]\left[Z_{2}\right] \\
& =\left[Z_{2}\right]^{+}[P]^{-}\left[P^{\prime}\right]\left[Z_{2}\right]
\end{aligned}
$$

where $[\bullet]^{+}$denotes Moore-Penrose general inverse(i.e., pseudo-inverse).

Combining (3) with (14)-(16), one can obtain

$$
[P]^{-}\left[P^{\prime}\right]=\operatorname{diag}\left[\frac{1}{A_{1} e^{j \varphi_{1}}}, \frac{1}{A_{1} e^{-j \varphi_{1}}}, 0, \cdots, 0\right]
$$

Therefore, the amplitude and the phase angle of the fundamental components can be obtained by computing the eigenvalues of square matrix $[I]^{+}[U]$.

\section{The Basic Principle of Fault Location}

The following Figure 1 shows a single phase transmis- 
sion system between two buses. A fault occurs at location $\mathrm{F}$ which is $x$ kilometer from bus $\mathrm{M}$, the voltage phasor at fault point $\dot{U}_{F}$ can be expressed as

$$
\left\{\begin{array}{c}
\dot{U}_{m F}=\dot{U}_{m} \operatorname{ch}(\gamma \cdot x)-Z_{c} \dot{I}_{m} \operatorname{sh}(\gamma \cdot x) \\
\dot{U}_{n F}=\left(\dot{U}_{n} \operatorname{ch}(\gamma \cdot(L-x))-Z_{c} \dot{I}_{n} \operatorname{sh}(\gamma \cdot(L-x))\right) e^{j \delta}
\end{array}\right.
$$

where $\dot{U}_{m}$ and $\dot{I}_{m}$ are the voltage and current at bus $\mathrm{M} ; \dot{U}_{n}$ and $I_{n}$ are the voltage and current at bus $\mathrm{N}$; $\gamma$ is wave propagation coefficient,

$$
\gamma=\sqrt{\left(R_{1}+j \omega L_{1}\right)\left(G_{1}+j \omega C_{1}\right)} ;
$$

$Z_{c}$ is surge impedance of transmission line,

$$
Z_{c}=\sqrt{\left(R_{1}+j \omega L_{1}\right) /\left(G_{1}+j \omega C_{1}\right)} ;
$$

$R_{1}, L_{1}, G_{1}$ and $C_{1}$ are resistance per kilometer, inductance per kilometer, conductance per kilometer, and capacitance per kilometer of transmission line respectively; $\mathrm{L}$ is the whole length of transmission line; $\delta$ is the asynchronous angle of sampling at both ends.

Theoretically, asynchronous data at both terminals only affect the phase of sinusoidal signal, but has no influence on the magnitudes; therefore the fault point voltage magnitudes measured from two ends are equal, i.e.

$$
\left|\dot{U}_{m F}\right|=\left|\dot{U}_{n F}\right|
$$

Substitution (18) into (19), one can obtain

$$
\begin{aligned}
& \left|\dot{U}_{m} \operatorname{ch}(\gamma x)-Z_{c} \dot{I}_{m} \operatorname{sh}(\gamma x)\right|= \\
& \left|\left(\dot{U}_{n} \operatorname{ch}(\gamma(L-x))-Z_{c} \dot{I}_{n} \operatorname{sh}(\gamma(L-x))\right)\right|
\end{aligned}
$$

Because the circuit parameters are known, and the electrical quantities of opposite end are also be obtained, so solving (20) can get the fault position $x$.

Also note that the voltage and current of (18)-(20) are decoupling modulus through phase-to-module transformation. The searching method for the fault location is as follows.

Assume $\varepsilon_{1}$ is a precision number which less than 1 , but close to 1 , and $\varepsilon_{2}$ is also a precision number which greater than 1, but close to1. Firstly, the transmission line is divided into $\mathrm{n}$ sections. If $\mathrm{n}$ is even number, then the initial iterative location $x$ is equal to $L_{n / 2}$; if $\mathrm{n}$ is odd number, then the initial iterative location $x$ is equal to

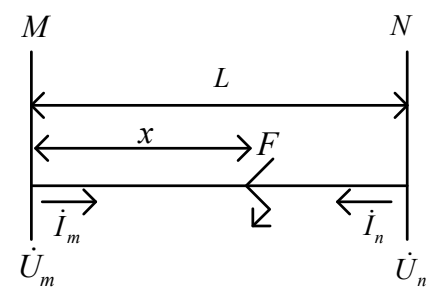

Figure 1. Single phase transmission system.
$L_{n+1 / 2}$. Substitution $x$ into (18), $\dot{U}_{m F}$ and $\dot{U}_{n F}$ can be computed respectively. If $\varepsilon_{1} \leq\left|\dot{U}_{m F} / \dot{U}_{n F}\right| \geq \varepsilon_{2}$, then $L_{n / 2}$ is the fault location; if $\left|\dot{U}_{m F} / \dot{U}_{n F}\right| \geq \varepsilon_{2}$, then the fault point locates in the left side of $L_{n / 2}$, so let $x$ equals to $L_{n / 2-1}$; if $\left|\dot{U}_{m F} / \dot{U}_{n F}\right| \leq \varepsilon_{1}$, then the fault point locates in the right side of $L_{n / 2}$, so let $x$ equals to $L_{n / 2+1}$. Substitution new value of $x$ into (18), and repeat the process. If $x=L_{k}$ and $\varepsilon_{2}<\left|\dot{U}_{m F} / \dot{U}_{n F}\right|$, then the fault point locates in the left side of $L_{k}$; if $x=L_{k-1}$, and $\left|\dot{U}_{m F} / \dot{U}_{n F}\right|<\varepsilon_{1}$, then the fault point locates in the right side of $L_{k-1}$. Thus it can be make sure that the fault location is located in $\mathrm{k} t h$ interval.

When the fault interval is determined, the fault interval can be subdivided to smaller intervals. Using (18), $\dot{U}_{m F}$ and $\dot{U}_{n F}$ of each point in the fault interval can be computed. If on some $x$ in fault interval, $\left|\frac{\dot{U}_{m F}}{\dot{U}_{n F}}\right|-1$ satisfies minimum in all of point, then $x$ is the fault location.

\section{Simulation and Verification}

In order to improve the fault location accuracy of twoterminal location algorithm during the transient process, the Fast Extraction method (FE method) is applied to two-terminal location algorithm. The demonstration project of Jindongnan-Jingmen 1000 kV UHV AC transmission has been simulated by ATP-EMTP software. The simulation model is shown in Figure 2. The total length is $654 \mathrm{~km}$. Positive sequence parameters of transmission line are $R_{1}=0.00758 \Omega / \mathrm{km}, X_{1}=0.26365 \Omega / \mathrm{km}$ and $C_{1}=0.01397 \mu \mathrm{F} / \mathrm{km}$. Zero sequence parameters of transmission line are $R_{0}=0.15421 \Omega / \mathrm{km}, X_{0}=0.8306 \Omega / \mathrm{km}$ and $C_{0}=0.00926 \mu \mathrm{F} / \mathrm{km}$. Jindongnan-Nanyang circuit is configured 2 groups of shunt reactors on the two terminal ends of the transmission line, whose capacity is 960 Mvar and 720 Mvar respectively. Nanyang-Jingmen circuit is configured 2 groups of shunt reactors on the two terminal ends of the transmission line, whose capacity is 720 Mvar and 600 Mvar respectively.

The Jindongnan-Nanyang transmission line is divided into 10 sections, fault location $K_{1}$ is located in every section end. There are five different fault types at different fault locations, which are single-line-to-ground fault, high-resistance single-line-to-ground fault with $500 \Omega$ transition resistance, phase-to-phase short circuit fault, phase-to-phase short circuit grounding fault, and threephase short circuit fault.

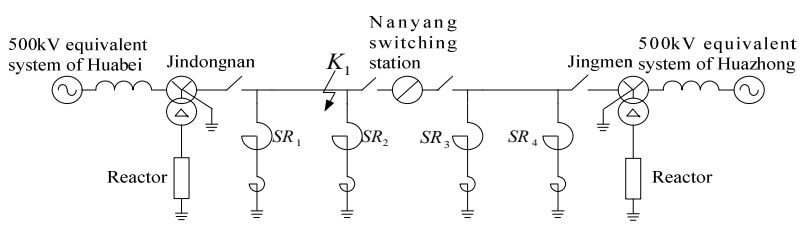

Figure 2. UHV AC demonstration project system. 
In practice, digital relays are equipped with analog low-pass antialiasing filters prior to the analog-to-digital converter. To accurately model the analog process of antialias filtering, the initial sampling rate in the simulations is set to $20 \mathrm{kHz}$. Then the voltage and current are passed through a second-order low-pass Butterworth filter with a 350-Hz cutoff frequency. The output of this low-pass filter is downsampled to $f_{s}=4 \mathrm{kHz}$. MATLAB is used to verify the location effect of Fast Extraction method, which is also compared with the measured location of Fourier algorithm.

Throughout the entire discussion, $D_{f}$ and L represent the real fault location and the whole length of transmission line. $D_{C C}$ and $D_{C P}$ represent the location measured by the conventional Fourier method and the proposed method, respectively. $\varepsilon_{A C}$ and $\varepsilon_{R C}$ represent the absolute error and the relative error of the fault location given by the conventional Fourier algorithm, respectively. $\varepsilon_{A P}$ and $\varepsilon_{R P}$ represent the absolute error and the relative error of the fault location given by the proposed scheme, respectively. These errors are defined as

$$
\begin{gathered}
\varepsilon_{A C}=D_{C C}-D_{f} \\
\varepsilon_{R C}=\frac{D_{C C}-D_{f}}{L} \times 100 \% \\
\varepsilon_{A P}=D_{C P}-D_{f}
\end{gathered}
$$

and

$$
\varepsilon_{R P}=\frac{D_{C P}-D_{f}}{L} \times 100 \%
$$

The following gives an example as the location effect of the conventional Fourier algorithm and the proposed method about single-line-to-ground fault at $326.7 \mathrm{~km}$ from Jindongnan to illustrate. Fault components of the voltage and the current are shown in Figure 3(a)-3(b). The magnitude and phase angle comparisons measured by the proposed method and one cycle Fourier algorithm are shown in Figure 3(c)-3(f). The Fast Extraction method can accurately compute the fundamental components of the voltage and the current, and filter out the influence of decaying DC offset and harmonics.

The fault location obtained by the positive-sequence fault components, the negative-sequence fault components, and the zero-sequence fault components measured by the conventional Fourier method and the proposed method are shown in Figures 4-6, respectively. The result of the proposed method is almost a straight line, but the result of the traditional Fourier algorithm is an upand-down curve.

Because different fault types all contain positive- sequence fault components, so the following location results are obtained by positive-sequence fault components. Tables 1-5 represent the location performance of the conventional Fourier method and the proposed method, respectively, in terms of error in the measurement of absolute error and relative error for different fault types such as single-line-to-ground fault, high-resistance single-line-to-ground with 500 transition resistance, phaseto-phase short circuit fault, phase-to-phase short circuit grounding fault and three-phase short circuit fault.

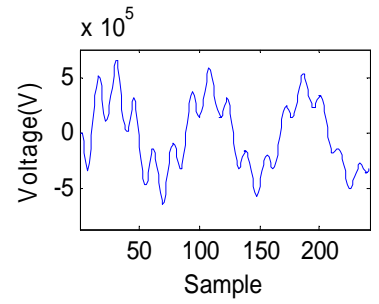

(a)

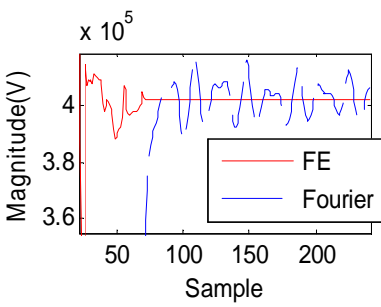

(c)

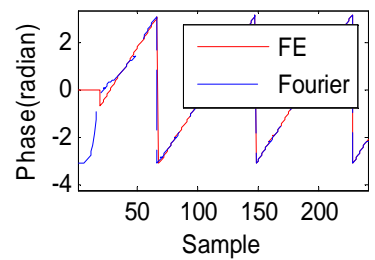

(e)

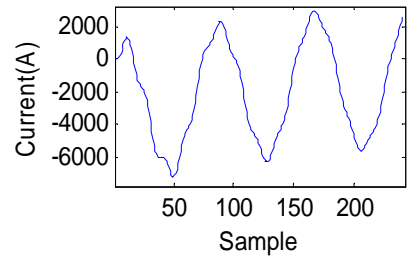

(b)

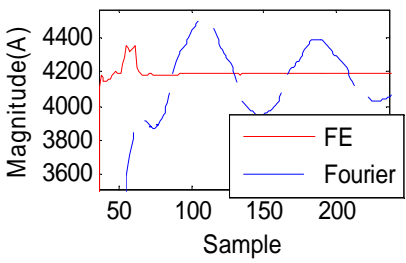

(d)

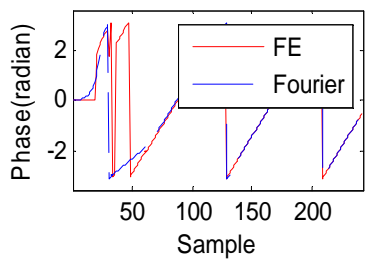

(f)
Figure 3. Fault components of the voltage and the current under single-line-to-ground fault and the magnitude and phase angle comparisons extracted by the Fast Extraction method and one cycle Fourier algorithm.

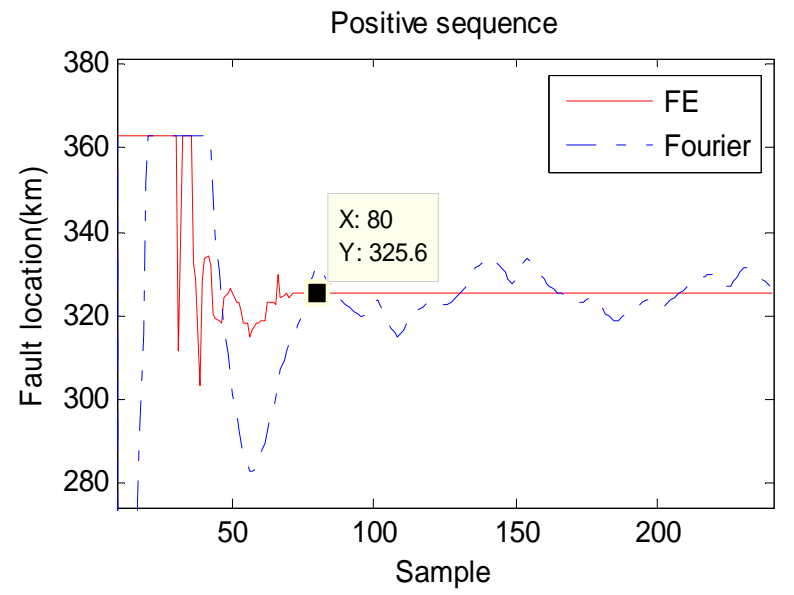

Figure 4. $D_{C C}$ and $D_{C P}$ measured by positive-sequence fault components. 


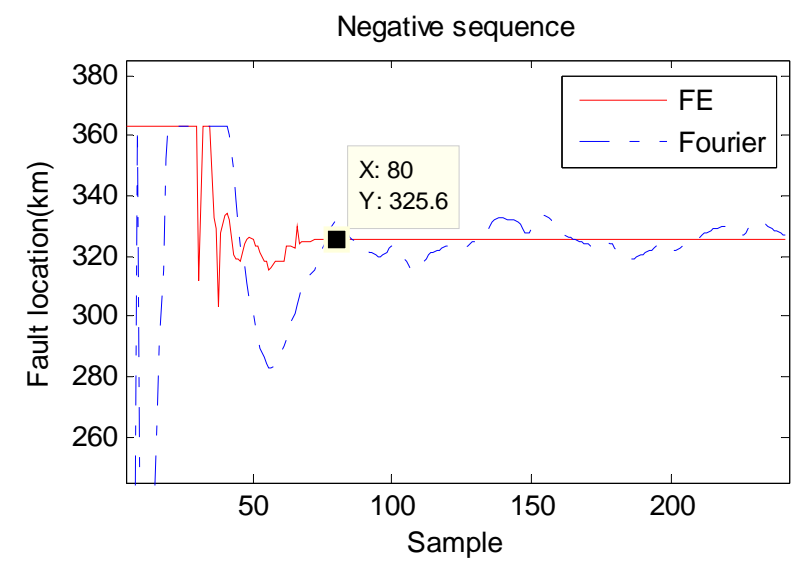

Figure 5. $D_{C C}$ and $D_{C P}$ measured by negative-sequence fault components.

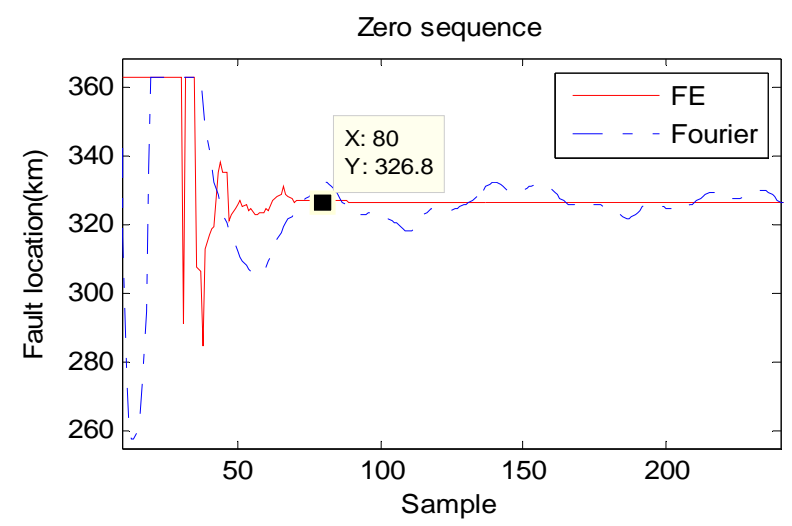

Figure 6. $D_{C C}$ and $D_{C P}$ measured by zero-sequence fault components.

Table 1. Location results of single-line-to-ground fault using positive sequence fault fundamental components computed by Fast Extraction method and one cycle Fourier algorithm.

\begin{tabular}{ccccc}
\hline$D_{f}(\mathrm{~km})$ & $D_{C C}(\mathrm{~km})$ & $\varepsilon_{R C}(\mathbf{\%})$ & $D_{C P}(\mathrm{~km})$ & $\varepsilon_{R P} \mathbf{( \% )}$ \\
\hline 0 & {$[0.0,11.7]$} & {$[0.00,3.22]$} & {$[1.30,1.60]$} & {$[0.36,0.44]$} \\
36.3 & {$[30.4,46.7]$} & {$[-1.63,2.87]$} & {$[37.3,37.5]$} & {$[0.28,0.33]$} \\
72.6 & {$[65.0,84.0]$} & {$[-2.09,3.14]$} & {$[73.2,73.5]$} & {$[0.17,0.25]$} \\
108.9 & {$[102.2,117.8]$} & {$[-1.85,2.45]$} & {$[109.2,109.4]$} & {$[0.08,0.14]$} \\
145.2 & {$[140.3,151.3]$} & {$[-1.35,1.68]$} & {$[145.2,145.3]$} & {$[0.00,0.027]$} \\
181.5 & {$[180.9,181.5]$} & {$[-0.17,0.00]$} & {$[181.1,181.2]$} & {$[-0.11,-0.08]$} \\
217.8 & {$[211.4,221.6]$} & {$[-1.76,-1.05]$} & {$[217.1,217.1]$} & {$[-0.19,-0.17]$} \\
254.1 & {$[244.8,260.5]$} & {$[-2.56,1.76]$} & {$[253.0,253.1]$} & {$[-0.30,-0.28]$} \\
290.4 & {$[278.8,297.9]$} & {$[-3.20,2.07]$} & {$[288.9,289.0]$} & {$[-0.41,-0.39]$} \\
326.7 & {$[315.2,333.6]$} & {$[-3.17,1.9]$} & {$[325.4,325.6]$} & {$[-0.36,-0.30]$} \\
363 & {$[349.9,363.0]$} & {$[-3.61,0.0]$} & {$[361.5,361.6]$} & {$[-0.41,-0.39]$} \\
\hline
\end{tabular}

Table 2. Location results of high resistance single-line-toground fault with $500 \Omega$ using positive sequence fault fundamental components computed by Fast Extraction method and one cycle Fourier algorithm.

\begin{tabular}{ccccc}
\hline$D_{f}(\mathrm{~km})$ & $D_{C C}(\mathrm{~km})$ & $\varepsilon_{R C} \mathbf{( \% )}$ & $D_{C P}(\mathrm{~km})$ & $\varepsilon_{R P} \mathbf{( \% )}$ \\
\hline 0 & {$[0.00,17.9]$} & {$[0,4.93]$} & {$[1.40,1.60]$} & {$[0.39,0.44]$} \\
36.3 & {$[34.4,51.0]$} & {$[-0.52,4.05]$} & {$[37.3,37.6]$} & {$[0.28,0.36]$} \\
72.6 & {$[70.3,83.9]$} & {$[-0.63,3.11]$} & {$[73.2,73.5]$} & {$[0.17,0.25]$} \\
108.9 & {$[105.7,116.8]$} & {$[-0.88,2.18]$} & {$[109.2,109.3]$} & {$[0.08,0.11]$} \\
145.2 & {$[141.8,149.9]$} & {$[-0.94,1.29]$} & {$[145.2,145.3]$} & {$[0.00,0.027]$} \\
181.5 & {$[181.1,181.3]$} & {$[-0.11,-0.06]$} & {$[181.1,181.2]$} & {$[-0.11,-0.08]$} \\
217.8 & {$[212.4,220.4]$} & {$[-1.49,0.72]$} & {$[217.0,217.1]$} & {$[-0.22,-0.19]$} \\
254.1 & {$[245.7,256.6]$} & {$[-2.31,0.69]$} & {$[252.9,253.1]$} & {$[-0.33,-0.28]$} \\
290.4 & {$[278.6,291.9]$} & {$[-3.25,0.41]$} & {$[288.9,289.0]$} & {$[-0.41,-0.39]$} \\
326.7 & {$[312.2,328.7]$} & {$[-3.99,0.55]$} & {$[325.4,325.7]$} & {$[-0.36,-0.28]$} \\
363 & {$[345.2,363.0]$} & {$[-4.90,0.00]$} & {$[361.5,361.6]$} & {$[-0.41,-0.39]$} \\
\hline
\end{tabular}

Table 3. Location results of phase-to-phase short circuit fault using positive sequence fault fundamental components computed by Fast Extraction method and one cycle Fourier algorithm.

\begin{tabular}{ccccc}
\hline$D_{f}(\mathrm{~km})$ & $D_{C C}(\mathrm{~km})$ & $\varepsilon_{R C} \mathbf{( \% )}$ & $D_{C P}(\mathrm{~km})$ & $\varepsilon_{R P}(\mathbf{\%})$ \\
\hline 0 & {$[0.0,9.2]$} & {$[0,2.53]$} & 1.5 & 0.41 \\
36.3 & {$[29.9,45.2]$} & {$[-1.76,2.45]$} & 37.4 & 0.30 \\
72.6 & {$[67.6,79.3]$} & {$[-1.38,1.85]$} & 73.4 & 0.22 \\
108.9 & {$[106.4,112.3]$} & {$[-0.69,0.94]$} & 109.3 & 0.11 \\
145.2 & {$[143.3,147.1]$} & {$[-0.52,0.52]$} & 145.2 & 0 \\
181.5 & {$[180.8,181.5]$} & {$[-0.19,0.00]$} & 181.2 & -0.08 \\
217.8 & {$[215.7,218.6]$} & {$[-0.58,0.22]$} & 217.1 & -0.19 \\
254.1 & {$[250.5,255.5]$} & {$[-0.99,0.39]$} & 253 & -0.30 \\
290.4 & {$[283.5,294.4]$} & {$[-1.90,1.10]$} & {$[288.9,289]$} & {$[-0.41,-0.39]$} \\
326.7 & {$[312.8,332.8]$} & {$[-3.83,1.68]$} & 325.6 & -0.30 \\
363 & {$[354.2,363.0]$} & {$[-2.42,0.00]$} & 361.5 & -0.41 \\
\hline
\end{tabular}


Table 4. Location results of phase-to-phase short circuit grounding faultusing positive sequence fault fundamental components computed by Fast Extraction method and one cycle Fourier algorithm .

\begin{tabular}{ccccc}
\hline$D_{f}(\mathrm{~km})$ & $D_{C C}(\mathrm{~km})$ & $\varepsilon_{R C}(\%)$ & $D_{C P}(\mathrm{~km})$ & $\varepsilon_{R P}(\%)$ \\
\hline 0 & {$[0,8.2]$} & {$[0,2.25]$} & {$[1.5,1.7]$} & {$[0.41,0.47]$} \\
36.3 & {$[31.8,43.8]$} & {$[-1.24,2.1]$} & 37.4 & 0.3 \\
72.6 & {$[69.1,78.4]$} & {$[-0.96,1.59]$} & 73.4 & 0.22 \\
108.9 & {$[107,111.8]$} & {$[-0.52,0.79]$} & {$[109.2,109.4]$} & {$[0.08,0.14]$} \\
145.2 & {$[143.7,147.1]$} & {$[-0.41,0.52]$} & 145.2 & 0 \\
181.5 & {$[180.9,181.4]$} & {$[-0.17,-0.03]$} & 181.2 & -0.08 \\
217.8 & {$[215.7,218.6]$} & {$[-0.58,0.25]$} & 217.1 & -0.19 \\
254.1 & {$[250.9,255.0]$} & {$[-0.88,0.25]$} & 253 & -0.30 \\
290.4 & {$[284.2,293.0]$} & {$[-1.71,0.72]$} & {$[288.9,289]$} & {$[-0.41,0.72]$} \\
326.7 & {$[319.5,331.1]$} & {$[-1.98,1.21]$} & 325.6 & -0.30 \\
363 & {$[354.9,363.0]$} & {$[-2.23,0.00]$} & {$[361.3,361.6]$} & {$[-0.47,-0.39]$} \\
\hline
\end{tabular}

Table 5. Location results of three-phase short circuit fault using positive sequence fault fundamental components computed by Fast Extraction method and one cycle Fourier algorithm.

\begin{tabular}{ccccc}
\hline$D_{f}(\mathrm{~km})$ & $D_{C C}(\mathrm{~km})$ & $\varepsilon_{R C}(\%)$ & $D_{C P}(\mathrm{~km})$ & $\varepsilon_{R P}(\%)$ \\
\hline 0 & {$[0.0,8.2]$} & {$[0.0,2.26]$} & 1.5 & 0.41 \\
36.3 & {$[29.6,45.8]$} & {$[-1.85,2.62]$} & 37.4 & 0.30 \\
72.6 & {$[67.4,79.7]$} & {$[-1.43,1.96]$} & 73.4 & 0.22 \\
108.9 & {$[107.5,111.2]$} & {$[-0.39,0.63]$} & 109.3 & 0.11 \\
145.2 & {$[143.3,147.8]$} & {$[-0.52,0.72]$} & 145.2 & 0 \\
181.5 & {$[180.7,181.6]$} & {$[-0.22,0.027]$} & 181.2 & 0.08 \\
217.8 & {$[213.7,219.2]$} & {$[-1.13,0.39]$} & 217.1 & -0.19 \\
254.1 & {$[251.1,254.7]$} & {$[-0.83,0.17]$} & 253 & -0.30 \\
290.4 & {$[282.6,295.0]$} & {$[-2.15,1.27]$} & {$[288.9,289]$} & {$[-0.41,-0.39]$} \\
326.7 & {$[317.1,333,4]$} & {$[-2.64,1.85]$} & 325.6 & -0.30 \\
363 & {$[355.0,363.0]$} & {$[-2.20,0.00]$} & 361.5 & -0.41 \\
\hline
\end{tabular}

From the above Tables 1-5, it is to be noted that the maximum relative error of fault location given by the conventional Fourier algorithm is in a limit of [-4.9\%, $4.05 \%$ ], but the maximum relative error of fault location given by the proposed method remains within a limit of $\pm 0.47 \%$. The relative error given by the proposed method is smaller one order of magnitude than that of the conventional Fourier method. So the precision of two-terminal fault location given by the proposed method is more accurate than that of Fourier method.

\section{Conclusions}

During the transient process of UHV transmission line, voltage and current contain lots of decaying DC offset and harmonics. The phasor precision given by the existing one-cycle Fourier algorithm is lower. In order to improve the precision, this paper introduces a Fast Extraction method to compute the fundamental components of the fault components about voltage and current.

The distributed parameter of transmission line is simulated by ATP-EMTP software. And the location results based on the fault components of voltage and current obtained by the proposed method is compared with that of the conventional Fourier algorithm. The simulation results show that the proposed method can efficiently improve the precision of fault location during the transient process, and makes the error of location results less than $0.5 \%$; therefore it has a good application prospect.

\section{REFERENCES}

[1] Z. M. Du and F. Zhao, "A New Fault Location Algorithm Using Asynchronized Two-terminal Data,” High Voltage Engineering, Vol. 29, No. 11, 2003, pp. 11-12.

[2] D. C. Liu, X. W. Du, Y. Li and Y. Huang, "Fault Location Using Two-terminal Data for HV\& Long Transmission Line Based on Genetic Algorithm," High Voltage Engineering, Vol. 33,No. 3, 2007, pp. 21-25.

[3] Z. Chen, X. Z. Dong and C. M. Luo, “A New Accurate Two-terminal Location Algorithm for Series Compensated Line," Proceedings of the CSEE, Vol. 23, No. 1, 2003, pp. 11-15.

[4] L. Teng, W. S. Liu, Y. Li, G. C. Li and H. X. Qin, “A Novel Practical Accurate Fault Location Algorithm for HV Transmission Line," Automation of Electric Power Systems, Vol. 25, No.9, 2001, pp. 24-27.

[5] H. C. Shu, D. J. Si, Y. Z. Ge and X. Y. Chen, "Study on Practical Fault Location Algorithm for Two-terminal HV and EHV Transmission Lines Using Asynchronous Dada at Both Ends,” Power System Technology, Vol. 24, No. 2, 2000, pp. 45-49.

[6] Z. T. Xin, D. J. Shang and X. G. Yin, "False Root and Its Improvement of a Two-terminal Fault Location Algorithm on Transmission line,” Relay, Vol. 33, No. 6, 2005, pp. 36-39.

[7] G. X. Jia, K. C. Wang and J. L. He, "Method of Eliminating Attenuating DC Component for Extended Holocycle Fourier Algorithm," Automation of Electric Power Systems, Vol. 16, No. 2, 1992, pp. 14-17.

[8] Z. J. Zhang, H. Z. Wang and P. R. He, "An Improved Fourier Algorithm and Differential Equation Algorithm," Automation of Electric Power Systems, Vol. 7, No. 5, 1983, pp. 20-30.

[9] Y. Hua and T. K. Sarker, "Matrix Pencil Method and Its Performance," Acoustics, Speech, and Signal Processing, Vol. 4, 1988, pp. 2476-2479. 
[10] Y. Hua and T. K. Sarker, "Matrix Pencil Method for Estimating Parameters of Exponentially Damped/ undamped sinusoids in Noise," IEEE Transaction on Acoustics, Speech, and Signal Processing, Vol. 38, No. 5, 1990, pp. 814-824. doi:10.1109/29.56027

[11] Y. Hua and T. K. Sarker, "On SVD for Estimating Generalized Eigenvalues of Singular Matrix Pencil in Noise," IEEE Transaction on Signal Processing, Vol. 39, No. 4, 1991, pp. 892-900. ․ doi:10.1109/78.80911
[12] T. K. Sarker and O. Pereira, "Using the Matrix Pencil Method to Estimate the Parameters of a Sum of Complex Exponentials,” IEEE Antennas and Propagation Magazine, Vol. 37, No. 1, 1995, pp. 48-55. doi:10.1109/74.370583

[13] J. L. Suonan, B. Wang, L. Wang, J. F. Sun and M. Xiao, "A Fast Phasor Estimator for Power System," Proceedings of the CSEE, Vol. 33, No. 1, 2013, pp. 123-129. 